\section{REVISTA INTERNACIONAL DE CIENCIAS DEL DEPORTE} International Journal of Sport Science

Rev. int. cienc. deporte

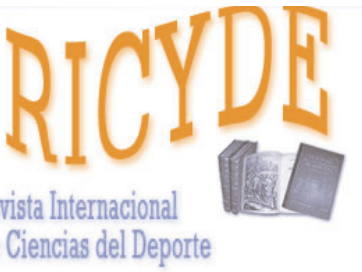

International Journal of Sport Science VOLUMEN IX - AÑO IX

Páginas:360-376 ISSN:1885-3137 No 34 - Octubre - 2013

\title{
Características del esfuerzo en competición en jugadoras de baloncesto de élite durante las fases finales de la Euroliga y el Campeonato del Mundo The competitive demands of elite female basketball during the play-offs of the Euroleague and World Championship
}

\author{
Laline Oliveira-Da-Silva \\ Universidad de León \\ Silvia Sedano-Campo \\ Universidad Europea Miguel de Cervantes. Valladolid \\ Juan Carlos Redondo-Castán \\ Universidad de León
}

\section{Resumen}

El objetivo principal del estudio fue definir las características del esfuerzo desde el punto de vista de la carga externa en jugadoras de baloncesto de alto nivel en dos competiciones: Euroliga femenina y Campeonato del Mundo. Para ello, se analizaron ocho partidos de las fases finales de ambas competiciones. Se llevó a cabo un análisis detallado de los patrones de movimiento de 96 jugadoras pertenecientes a ocho selecciones nacionales y a cinco equipos profesionales. Se utilizaron ocho categorías de actividad a la hora de clasificar las acciones y los desplazamientos en los partidos: parado/caminando, trote, carrera, sprint, salto y shuffle baja, media y alta intensidad. Se calculó el tiempo total en cancha, el tiempo real y el porcentaje de tiempo real respecto del tiempo total. Por otro lado se analizó la frecuencia y la duración media de cada actividad así como el porcentaje de tiempo real empleado en cada una de ellas. Los resultados obtenidos confirman la naturaleza híbrida del esfuerzo, en el que se alternan fases aeróbicas y anaeróbicas, predominando las primeras sobre las segundas. Se observa que los esfuerzos anaeróbicos son los que marcan la diferencia entre niveles competitivos y entre posiciones habituales de juego. Asimismo se observa que sólo las jugadoras en la posición de base presentan un perfil específico de esfuerzo que las diferencia de aleros y pívots.

Palabras clave: carga externa; desplazamientos; frecuencia; duración media; posición.

\section{Abstract}

The main aim of the current study was to establish the competitive demands, in terms of external load, of elite female basketball players during the final phase of Euroleague and World Championship. Eight matches corresponding to the play-offs of these competitions were analyzed. A detailed analysis of the movement patterns of 96 players was performed. Eight categories were employed to classify the movements: stand/walk, jog, run, stride/sprint, low shuffle, medium shuffle, high shuffle and jump. Total time in court, live time and percentage of live time over total time were calculated. On the other hand, the frequency and the mean duration of each movement category were also measured. Results illustrate the intermittent nature of female basketball. The majority of time appears to be devoted to activities more aerobic in nature, and only a small percentage of time is spent engaged in high-intensity activity. However, anaerobic capacity seems to be a better predictor of basketball performance. On the other hand, only guards seem to have a specific movement pattern different from that of centers or forwards.

Key words: external load; movements; frequency; mean duration; playing position.

Correspondencia/correspondence: Silvia Sedano Campo

Laboratorio de Fisiología. Universidad Europea Miguel de Cervantes. Valladolid. España

Email: ssedano@uemc.es 
Oliveira-Da-Silva, L.; Sedano-Campo, S.; Redondo-Castán, J.C. (2013). Características del esfuerzo en competición en jugadoras de baloncesto de élite durante las fases finales de la Euroliga y el Campeonato del Mundo. RICYDE. Rev. int. cienc. deporte. 34(9), 360-376. http://dx.doi.org/10.5232/ricyde2013.03405

\section{Introducción}

$\mathrm{T}$ eniendo en cuenta el número actual de practicantes, el baloncesto puede considerarse uno de los deportes más populares del mundo, de ahí que la comunidad científica haya mostrado un especial interés a la hora de realizar estudios referentes a los factores de rendimiento y a la carga fisiológica que implica esta modalidad deportiva. El baloncesto se compone de habilidades técnico-tácticas complejas que tienen una influencia directa en los requerimientos fisiológicos impuestos sobre el jugador durante la competición (Drinkwater, Pyne y McKenna, 2008; Ziv y Lidor., 2009). Son muchos los estudios que consideran que tiene un carácter híbrido, en el que la mayor cantidad de energía movilizada proviene de la vía aeróbica (Abdelkrim, El Fazaa y El Ati, 2007; McInnes, Carlson, Jones y McKenna, 1995; Narazaki, Berg, Stergiou y Chen, 2009); sin embargo, al igual que ocurre en otros deportes colectivos, las acciones de carácter explosivo, tales como cambios de dirección, saltos o desplazamientos a la máxima intensidad, así como las acciones específicas de juego como los tiros, los desmarques o los driblings, dependen de la vía anaeróbica y son determinantes en el rendimiento final de los deportistas (Chaouachi y col., 2009; McInnes y col., 1995; Narazaki y Berg, 2006; Ostojic, Mazic y Dikic, 2006). Así, Sallet, Perrier, Ferret, Vitelli y Baverel, (2005) efectuaron un estudio con jugadores franceses que confirma lo anterior, ya que concluye que la capacidad anaeróbica puede considerarse uno de los factores de rendimiento más importantes en esta modalidad deportiva, independientemente de que cuantitativamente la vía aeróbica goce de una mayor importancia en el suministro energético.

Históricamente, no sólo en baloncesto sino en todos los deportes colectivos, uno de los métodos más empleados para conocer esas características del esfuerzo ha sido y es la metodología observacional a través del análisis de video (Abdelkrim y col., 2007; Boyle, Mahoney y Wallace, 1994; McInnes y col., 1995; Taylor, 2003; Tessitore y col., 2006). En el momento actual, el carácter científico de la metodología observacional, contrastado por múltiples autores (Anguera, 1990; Medina y Delgado, 1999) nos lleva a pensar en ella como una buena herramienta para utilizar en el baloncesto femenino, que al igual que otros deportes colectivos cumple con los requisitos necesarios (Gil y Contreras, 2008): espontaneidad de los comportamientos, el juego se desarrolla en su contexto natural y permite la utilización de la tecnología para acceder con precisión a las conductas.

En general, se puede decir que las características del esfuerzo han sido ampliamente analizadas en baloncesto masculino, utilizando para ello estudios que evalúan los patrones de movimiento o las respuestas fisiológicas del deportista durante la competición, especialmente en términos de frecuencia cardiaca o de acumulación de lactato (Abdelkrim y col., 2007; Castagna y col., 2005; Miller y Barlett, 1994). Uno de los trabajos que marca un antes y un después en el análisis de las características del esfuerzo en baloncesto es el de McInnes y col. (1995). Estos autores analizan los patrones de movimiento de ocho jugadores profesionales distinguiendo varias categorías de movimiento perfectamente definidas, su frecuencia así como su duración media, llegando a la conclusión de que el patrón de movimiento de cada jugador está íntimamente relacionado con los requerimientos fisiológicos en términos de frecuencia cardiaca y acumulación de lactato. Se muestra por tanto una estrecha relación entre la carga interna y la carga externa tal y como ya habían señalado con anterioridad Hoffman y Maresh (2000). Además concluyen que esa demanda fisiológica es diferente en función del nivel competitivo y la posición habitual en el terreno de juego. Por su parte, Rodríguez-Alonso, Fernández-García , Pérez-Landaluce y Terrados (2003) también afirman que las demandas fisiológicas en baloncesto femenino son diferentes en función del nivel competitivo y de la posición habitual en la cancha. De hecho, al analizar la carga interna de la 
Oliveira-Da-Silva, L.; Sedano-Campo, S.; Redondo-Castán, J.C. (2013). Características del esfuerzo en competición en jugadoras de baloncesto de élite durante las fases finales de la Euroliga y el Campeonato del Mundo. RICYDE. Rev. int. cienc. deporte. 34(9), 360-376. http://dx.doi.org/10.5232/ricyde2013.03405

competición se observan valores diferentes tanto en la frecuencia cardiaca media como en el nivel medio de acumulación de lactato.

A pesar de que en la literatura analizada encontramos multitud de datos acerca de las características del esfuerzo en baloncesto masculino, tanto en términos de carga interna como de carga externa, no ocurre lo mismo en el caso del baloncesto femenino. En realidad se sabe más bien poco acerca de las demandas fisiológicas impuestas sobre las jugadoras de baloncesto en la competición, lo que según ciertos autores supone un problema a la hora de respetar algunos de los principios que han de marcar el entrenamiento deportivo, los de individualidad y especificidad (Bompa y Haff, 2009; Boyle y col., 1994; Naclerio, 2011). Trabajos como los de Rodríguez-Alonso y col. (2003) aportan algunos datos acerca de las exigencias competitivas, relacionando éstos con las características del nivel competitivo. Dichos datos pueden considerarse valiosos desde el punto de vista de la preparación física de las jugadoras y de la mejora del rendimiento, en un contexto en el que el tiempo disponible para la preparación cada vez es menor si bien las demandas profesionales cada vez más intensas (Drinkwater y col., 2008).

Teniendo en cuenta esa escasez de datos referentes a los patrones de movimiento en baloncesto femenino, el objetivo principal del presente estudio es definir las características del esfuerzo desde el punto de vista de la carga externa en jugadoras de baloncesto de alto nivel, para establecer valores de referencia que ayuden a entrenadores y preparadores físicos a definir el proceso de entrenamiento. Además, se pretende comparar la carga externa en dos de las principales competiciones femeninas internacionales, la Euroliga Femenina y el Campeonato del Mundo de selecciones. Otro de los objetivos del presente estudio es establecer los patrones de movimiento distinguiendo por posiciones habituales en la cancha. Teniendo en cuenta los objetivos anteriormente definidos, planteamos por un lado la hipótesis de que el esfuerzo en baloncesto femenino sigue un patrón híbrido en lo que hace referencia al suministro energético aeróbico-anaeróbico. Por otro lado, también planteamos la hipótesis de que existen diferencias en los patrones de movimiento tanto entre ambas competiciones como entre las distintas posiciones de juego.

\section{Método}

\section{Participantes}

Un total de 96 jugadoras de baloncesto pertenecientes a ocho selecciones nacionales y a cinco equipos profesionales fueron analizadas durante las fases finales de la Euroliga Femenina 2010 (EF) y del Campeonato del Mundo Femenino 2010 (CM). La muestra total de jugadoras estaba compuesta por 22 bases, 44 pívots y 30 aleros. En cada partido, se seleccionó a las 12 jugadoras que habían disputado más minutos (seis de cada equipo) para llevar a cabo un análisis detallado de los patrones de movimiento de las tres principales posiciones de este deporte. Se seleccionaron aquellas jugadoras que habían disputado más minutos con el objetivo de que los datos registrados y analizados fueran representativos del esfuerzo. Las características generales de estas jugadoras, detalladas en la tabla 1, se obtuvieron de la base de datos de la Federación Internacional de Baloncesto (FIBA). Todos los procedimientos descritos en el presente artículo fueron aprobados por el Comité Ético de la Universidad Europea Miguel de Cervantes. 
Oliveira-Da-Silva, L.; Sedano-Campo, S.; Redondo-Castán, J.C. (2013). Características del esfuerzo en competición en jugadoras de baloncesto de élite durante las fases finales de la Euroliga y el Campeonato del Mundo. RICYDE. Rev. int. cienc. deporte. 34(9), 360-376. http://dx.doi.org/10.5232/ricyde2013.03405

Tabla 1: Características generales de las jugadoras que componen la muestra objeto de estudio.

\begin{tabular}{|c|c|c|c|c|}
\hline Competición & $\mathrm{N}$ & Edad (años) & Talla $(\mathrm{cm})$ & Peso corporal $(\mathrm{kg})$ \\
\hline Euroliga Femenina (EF) & 48 & $27.21 \pm 4.63$ & $185.65 \pm 13.73$ & $78.64 \pm 10.41$ \\
\hline Campeonato del Mundo (CM) & 48 & $28.42 \pm 4.32$ & $177.55 \pm 11.88$ & $73.20 \pm 20.84$ \\
\hline Total & 96 & $27.81 \pm 4.43$ & $181.60 \pm 5.65$ & $75.92 \pm 15.67$ \\
\hline
\end{tabular}

\section{Procedimiento}

\section{$\underline{\text { Partidos analizados }}$}

Se analizaron en total cuatro partidos de la fase final de la EF (48 jugadoras) y cuatro del CM (48 jugadoras). Cada partido estaba compuesto por cuatro cuartos de 10 minutos de duración, con un descanso de 10 minutos entre el segundo y el tercer cuarto y otro descanso de dos minutos entre el primer y el segundo cuarto y entre el tercero y el cuarto. Los datos referentes a dichos partidos se encuentran recogidos en la tabla 2.

Tabla 2. Partidos analizados en el estudio.

\begin{tabular}{|c|c|c|c|}
\hline & Competición & Partido & Resultado \\
\hline 1 & $\begin{array}{l}\text { Campeonato del } \\
\text { Mundo (Final) }\end{array}$ & EEUU vs República Checa & $89-69$ \\
\hline 2 & $\begin{array}{l}\text { Campeonato del } \\
\text { Mundo }\left(3^{\text {er }} \text { y } 4^{\circ}\right. \\
\text { puesto })\end{array}$ & España vs Bielorrusia & $77-68$ \\
\hline 3 & $\begin{array}{l}\text { Campeonato del } \\
\text { Mundo (Semifinal) }\end{array}$ & República Checa vs Bielorrusia & $81-77$ \\
\hline 4 & $\begin{array}{l}\text { Campeonato del } \\
\text { Mundo (Semifinal) }\end{array}$ & EEUU vs España & $106-70$ \\
\hline 5 & $\begin{array}{l}\text { Euroliga femenina } \\
\text { (Final) }\end{array}$ & Spartak vs Halcón Avenida & $59-68$ \\
\hline 6 & $\begin{array}{l}\text { Euroliga femenina } \\
\text { (3er y } 4^{\circ} \text { puesto) }\end{array}$ & UMMC Ekaterinburg vs Ros Casares & $64-52$ \\
\hline 7 & $\begin{array}{l}\text { Euroliga femenina } \\
\text { (Semifinal) }\end{array}$ & Ros Casares vs Halcón Avenida & $49-61$ \\
\hline 8 & $\begin{array}{c}\text { Euroliga femenina } \\
\text { (Semifinal) }\end{array}$ & Spartak vs UMMC Ekaterinburg & $54-43$ \\
\hline
\end{tabular}

\section{Análisis tiempo-movimiento}

Las grabaciones empleadas para el estudio fueron proporcionadas por la FIBA y almacenadas en un ordenador portátil Sony Vaio 19.5 (PCG71312M Windows 7). Las filmaciones fueron analizadas fotograma a fotograma con una precisión de $0.04 \mathrm{~s}$ utilizando el software Adobe 
Oliveira-Da-Silva, L.; Sedano-Campo, S.; Redondo-Castán, J.C. (2013). Características del esfuerzo en competición en jugadoras de baloncesto de élite durante las fases finales de la Euroliga y el Campeonato del Mundo. RICYDE. Rev. int. cienc. deporte. 34(9), 360-376. http://dx.doi.org/10.5232/ricyde2013.03405

Premier 5.0 y volcando los datos en una planilla de observación diseñada ad hoc para el estudio. Cada secuencia de vídeo podía verse hacia delante y hacia atrás a diferentes velocidades, consiguiéndose de esa manera una mayor facilidad y precisión para el análisis de los movimientos. Los observadores fueron seleccionados tras un período de instrucción y de control de la calidad del dato para evitar incorrecciones y sesgos en los análisis, siguiendo la metodología planteada por Anguera, Blanco-Villaseñor, Losada y Sánchez Algarra (1999). La fiabilidad del análisis tiempo-movimiento se evaluó analizando la concordancia intra e interobservador mediante el coeficiente de correlación intraclase (ICC). Para evaluar la concordancia interobservador se compararon los datos obtenidos por cada uno de los observadores con el modelo de referencia del observador principal, que visionó el mismo partido para la misma jugadora en dos ocasiones. Para evaluar la concordancia intraobservador se compararon los datos registrados por el mismo evaluador en dos visionados de la misma jugadora en el mismo partido con diez días de separación entre ellos. El ICC en todos los casos y para todas las variables se situó entre 0.93 y 0.99 , por lo que se garantiza la fiabilidad de los datos empleados en el estudio.

Siguiendo la metodología planteada por McInnes y col. (1995) se utilizaron las siguientes categorías de actividad a la hora de clasificar las acciones y los desplazamientos en los partidos de baloncesto, categorías que pueden dividirse de manera global en acciones de baja intensidad o aeróbicas y de alta intensidad o anaeróbicas.

Acciones de baja intensidad:

1. Parado/caminando (stand/walk): Actividad de una intensidad no superior a la de caminar. En esta categoría, no se hace distinción entre permanecer parado de pie o caminar o entre diferentes intensidades de marcha. Parado/caminando también incluye aquellas situaciones en las que la jugadora está en posición defensiva pero sin movimiento.

2. Trote (jog): Movimiento con desplazamiento hacia delante o hacia atrás a una intensidad superior a la de la marcha pero sin alcanzar una alta velocidad.

3. Carrera (run): Movimiento con desplazamiento hacia delante o hacia atrás a una intensidad superior a la del trote pero sin llegar a alcanzar la alta intensidad correspondiente a la categoría de Sprint.

4. Shuffle baja intensidad (low shuffle): Desplazamiento generalmente lateral o hacia atrás en el que se produce un movimiento alternativo y cruzado de los pies. Se efectúa a baja velocidad, con poca frecuencia de movimiento de pies y con el tronco en posición erecta.

5. Shuffle media intensidad (medium shuffle): Movimiento similar al anterior pero con una velocidad más elevada pero no máxima.

Acciones de alta intensidad:

1. Sprint (stride/Sprint): Movimiento con desplazamiento hacia delante con una intensidad máxima o próxima al máximo.

2. Shuffle alta intensidad (high shuffle): Desplazamiento lateral con alta intensidad caracterizado por una alta velocidad de movimiento alternativo y cruzado de los pies en posición de flexión de rodillas.

3. Salto (jump): Movimiento desde el inicio de un salto hasta el momento de aterrizaje.

Con los datos registrados, se calcularon en primer lugar el tiempo total en cancha (TT), el tiempo real (TR) y el porcentaje de TR respecto del TT (TR/TT) para cada una de las 
Oliveira-Da-Silva, L.; Sedano-Campo, S.; Redondo-Castán, J.C. (2013). Características del esfuerzo en competición en jugadoras de baloncesto de élite durante las fases finales de la Euroliga y el Campeonato del Mundo. RICYDE. Rev. int. cienc. deporte. 34(9), 360-376. http://dx.doi.org/10.5232/ricyde2013.03405

competiciones y para el baloncesto femenino en su conjunto. El TT se refiere al total del tiempo del partido, incluyendo interrupciones como las provocadas por la salida del balón fuera de la cancha, los tiempos muertos o la ejecución de tiros libres. Sin embargo, no se incluyen los descansos entre cuartos o el tiempo en el que la jugadora es sustituida. Por el contrario, el TR se refiere sólo a los momentos del partido en los que el reloj está activado ya que el balón está en juego.

En segundo lugar y teniendo en cuenta la clasificación de las categorías de actividad anteriormente detallada, se calculó la frecuencia y la duración media de cada actividad así como el porcentaje de TR empleado en cada una de ellas. Se efectuó un análisis comparativo por competiciones (EF y CM) y por posiciones habituales de juego (bases, aleros y pívots) en ambas competiciones y en el baloncesto femenino en general.

\section{Análisis estadístico}

Los datos se expresan como media \pm desviación estándar y fueron analizados utilizando el software SPSS (v. 17). Para comparar el TT, TR y porcentaje TR/TT por competiciones se empleó el análisis de varianza de un solo factor (ANOVA). Por otro lado, para efectuar los distintos análisis comparativos entre competiciones y entre posiciones habituales de juego también se utilizó ANOVA. Si por medio de este procedimiento se detectaban diferencias significativas se llevaban a cabo las pruebas post-hoc de Bonferroni con el objetivo de localizarlas. El nivel de significación es estableció en $p<0.05$.

\section{Resultados}

Análisis comparativo en función de la competición

Los datos relativos al TT, al TR así como al porcentaje TR/TT se recogen en la tabla 3 . Al efectuar el análisis comparativo, los resultados obtenidos con ANOVA revelan la existencia de diferencias significativas entre EF y CM tanto para el TR como para el porcentaje TR/TT, siendo ambos valores superiores en EF. Sin embargo, no se observan diferencias significativas en el caso de TT.

Tabla 3: Tiempo total en cancha, tiempo real y porcentaje tiempo real/tiempo total en cancha en cada una de las competiciones. Análisis comparativo entre Euroliga femenina y Campeonato del Mundo y datos globales de baloncesto.

\begin{tabular}{|c|c|c|c|}
\hline Competición & Tiempo total (s) & Tiempo real (s) & Tiempo real/Tiempo total (\%) \\
\hline Euroliga femenina & $4775 \pm 482$ & $2226 \pm 205$ & $46.61 \pm 1.42$ \\
\hline Campeonato del Mundo & $4225 \pm 501$ & $1802 \pm 367$ & $42.65 \pm 1.33$ \\
\hline$F$ & .15 & 3.72 & 8.32 \\
\hline$p$ & .67 & $.002^{\star}$ & $.005^{\star}$ \\
\hline Baloncesto femenino & $4500 \pm 491$ & $2014 \pm 436$ & $44.63 \pm 1.38$ \\
\hline
\end{tabular}

${ }^{*} p<0.05$ 
Oliveira-Da-Silva, L.; Sedano-Campo, S.; Redondo-Castán, J.C. (2013). Características del esfuerzo en competición en jugadoras de baloncesto de élite durante las fases finales de la Euroliga y el Campeonato del Mundo. RICYDE. Rev. int. cienc. deporte. 34(9), 360-376. http://dx.doi.org/10.5232/ricyde2013.03405

Las tablas 4, 5 y 6 muestran la frecuencia de las diferentes categorías de actividad, la duración media de las mismas así como el porcentaje de TR que éstas ocupan. Con relación a la frecuencia, el ANOVA reveló la existencia de diferencias significativas entre competiciones en la frecuencia total, siendo ésta superior en el caso de EF y en las categorías parado/caminando, carrera, sprint y shuffle alta intensidad. En la primera categoría, los registros son más elevados en $\mathrm{CM}$, mientras que en las categorías restantes, éstos son superiores en EF. En general se observa que la frecuencia de actividades de baja intensidad es superior (EF:1044 \pm 11.8 ; CM:972 \pm 16.7 ; Baloncesto femenino:1008 \pm 14.3 ) a la de las acciones de alta intensidad (EF:265 \pm 10.3 ; CM:126 \pm 12.7 ; Baloncesto femenino:195.5 \pm 11.5 ).

Por otro lado, la duración media de cada actividad es similar en ambas competiciones, siendo en todos los casos inferior a 2.3 segundos. ANOVA solo reveló la existencia de diferencias estadísticamente significativas para la categoría shuffle alta intensidad, siendo mayor la duración en el caso de EF.

En lo que hace referencia al porcentaje de TR empleado en cada categoría de actividad, ANOVA reveló la existencia de diferencias significativas entre competiciones en las categorías parado/caminando, shuffle baja intensidad, sprint y shuffle alta intensidad, siendo los registros superiores en CM para las dos primeras y en EF para las dos siguientes. Si analizamos los datos obtenidos en baloncesto femenino en su conjunto, podemos observar que el mayor porcentaje de tiempo lo ocupa la categoría parado/caminando, seguida de trote, carrera, shuffle baja intensidad y shuffle media intensidad. Los menores porcentajes los ocupan las categorías de sprint, shuffle alta intensidad y salto. Si atendemos en conjunto al porcentaje de TR ocupado por las acciones de baja intensidad y de alta intensidad observaremos que las primeras registran un valor muy superior tanto en EF (baja intensidad: 81\%; alta intensidad: $19 \%$ ) como en CM (baja intensidad:91\%; alta intensidad: 9\%) o en el baloncesto femenino en general (baja intensidad:85.8\%;alta intensidad:14.2\%). 
Oliveira-Da-Silva, L.; Sedano-Campo, S.; Redondo-Castán, J.C. (2013). Características del esfuerzo en competición en jugadoras de baloncesto de élite durante las fases finales de la Euroliga y el Campeonato del Mundo. RICYDE. Rev. int. cienc. deporte. 34(9), 360-376. http://dx.doi.org/10.5232/ricyde2013.03405

Tabla 4: Frecuencia de las diferentes categorías de actividad en cada competición y en baloncesto femenino en su conjunto.

\begin{tabular}{|c|c|c|c|c|c|c|c|c|c|}
\hline & \multicolumn{5}{|c|}{ Acciones de baja intensidad } & \multicolumn{3}{|c|}{ Acciones de alta intensidad } & \multirow[b]{2}{*}{ Total } \\
\hline Competición & $\begin{array}{c}\text { Parado/ } \\
\text { caminando }\end{array}$ & Trote & Carrera & Shuffle baja intensidad & $\begin{array}{c}\text { Shuffle media } \\
\text { intensidad }\end{array}$ & $\begin{array}{l}\text { Shuffle alta } \\
\text { intensidad }\end{array}$ & Sprint & Salto & \\
\hline Euroliga femenina & $198 \pm 9.21$ & $176 \pm 15.13$ & $231 \pm 16.15$ & $227 \pm 7.81$ & $212 \pm 11$ & $100 \pm 6.32$ & $141 \pm 21.36$ & $24 \pm 3.20$ & $1309 \pm 11.25$ \\
\hline Campeonato del Mundo & $225 \pm 11.22$ & $172 \pm 17.93$ & $177 \pm 21.13$ & $206 \pm 14.31$ & $192 \pm 19.11$ & $50 \pm 11.12$ & $54 \pm 17.97$ & $22 \pm 9.20$ & $1098 \pm 15.23$ \\
\hline$F$ & 7.28 & 1.46 & 5.11 & .11 & .65 & 8.22 & 3.61 & .73 & 6.34 \\
\hline$p$ & $.01^{*}$ & .89 & $.003^{*}$ & .77 & .82 & $.02^{*}$ & $.001^{\star}$ & .98 & $.01^{*}$ \\
\hline BALONCESTO FEMENINO & $211.52 \pm 10.21$ & $174 \pm 16.53$ & $204 \pm 18.64$ & $216.5 \pm 11.05$ & $202 \pm 15.05$ & $75 \pm 8.72$ & $97.5 \pm 19.66$ & $23 \pm 6.20$ & $1203.57 \pm 13.24$ \\
\hline
\end{tabular}

Tabla 5: Duración media (s) de las acciones en cada categoría de actividad en cada una de las competiciones y en baloncesto femenino.

\begin{tabular}{|c|c|c|c|c|c|c|c|c|c|}
\hline & \multicolumn{5}{|c|}{ Acciones de baja intensidad } & \multicolumn{3}{|c|}{ Acciones de alta intensidad } & \multirow[b]{2}{*}{ Media } \\
\hline Competición & $\begin{array}{c}\text { Parado/ } \\
\text { caminando }\end{array}$ & Trote & Carrera & $\begin{array}{l}\text { Shuffle baja } \\
\text { intensidad }\end{array}$ & $\begin{array}{c}\text { Shuffle media } \\
\text { intensidad }\end{array}$ & $\begin{array}{l}\text { Shuffle alta } \\
\text { intensidad }\end{array}$ & Sprint & Salto & \\
\hline Euroliga femenina & $2.10 \pm .10$ & $1.80 \pm .06$ & $1.71 \pm .12$ & $1.61 \pm .11$ & $1.80 \pm .09$ & $2.10 \pm .10$ & $1.50 \pm .07$ & $1.01 \pm .09$ & $1.70 \pm .09$ \\
\hline $\begin{array}{l}\text { Campeonato del } \\
\text { Mundo }\end{array}$ & $2.20 \pm .13$ & $1.80 \pm .08$ & $1.81 \pm .09$ & $1.71 \pm .06$ & $1.71 \pm .12$ & $1.70 \pm .11$ & $1.40 \pm .11$ & $.90 \pm .07$ & $1.64 \pm .09$ \\
\hline $\mathrm{F}$ & .32 & .68 & .22 & .49 & .55 & 7.24 & .22 & 1.11 & .23 \\
\hline$p$ & .91 & .74 & .79 & .67 & .98 & $.03^{*}$ & .69 & .14 & .69 \\
\hline $\begin{array}{l}\text { BALONCESTO } \\
\text { FEMENINO }\end{array}$ & $2.15 \pm .11$ & $1.80 \pm .07$ & $1.76 \pm .10$ & $1.65 \pm .08$ & $1.75 \pm .10$ & $1.90 \pm .10$ & $1.45 \pm .09$ & $.95 \pm .08$ & $1.67 \pm .09$ \\
\hline
\end{tabular}

Tabla 6: Porcentaje de Tiempo Real empleado en cada categoría de actividad en cada competición y en baloncesto femenino.

\begin{tabular}{|c|c|c|c|c|c|c|c|c|}
\hline & \multicolumn{5}{|c|}{ Acciones de baja intensidad } & \multicolumn{3}{|c|}{ Acciones de alta intensidad } \\
\hline Competición & $\begin{array}{c}\text { Parado/ } \\
\text { caminando }\end{array}$ & Trote & Carrera & $\begin{array}{l}\text { Shuffle baja } \\
\text { intensidad }\end{array}$ & $\begin{array}{l}\text { Shuffle media } \\
\text { intensidad }\end{array}$ & $\begin{array}{l}\text { Shuffle alta } \\
\text { intensidad }\end{array}$ & Sprint & Salto \\
\hline $\begin{array}{c}\text { Euroliga } \\
\text { femenina }\end{array}$ & $17.96 \pm 1.50$ & $13.68 \pm .80$ & $16.96 \pm .90$ & $15.68 \pm .80$ & $16.48 \pm .90$ & $9.07 \pm .75$ & $9.13 \pm 1.10$ & $1.03 \pm .50$ \\
\hline $\begin{array}{c}\text { Campeonato del } \\
\text { Mundo }\end{array}$ & $25 \pm 1.10$ & $15.63 \pm 1.10$ & $16.09 \pm .90$ & $17.68 \pm .75$ & $16.48 \pm 1.15$ & $4.34 \pm .60$ & $3.81 \pm .60$ & $1 \pm .90$ \\
\hline $\mathrm{F}$ & 3.61 & .22 & .41 & 4.35 & .41 & 5.22 & 7.25 & .22 \\
\hline$p$ & $.001^{*}$ & .87 & .93 & $.001^{\star}$ & .93 & $.002^{*}$ & $.001^{*}$ & .77 \\
\hline $\begin{array}{l}\text { Baloncesto } \\
\text { femenino }\end{array}$ & $21.48 \pm 1.30$ & $14.65 \pm .95$ & $16.52 \pm .90$ & $16.68 \pm .77$ & $16.48 \pm 1.02$ & $6.70 \pm .67$ & $6.47 \pm .85$ & $1.01 \pm .70$ \\
\hline
\end{tabular}


Oliveira-Da-Silva, L.; Sedano-Campo, S.; Redondo-Castán, J.C. (2013). Características del esfuerzo en competición en jugadoras de baloncesto de élite durante las fases finales de la Euroliga y el Campeonato del Mundo. RICYDE. Rev. int. cienc. deporte. 34(9), 360-376. http://dx.doi.org/10.5232/ricyde2013.03405

\section{Análisis comparativo en función de la posición habitual de juego}

En la tabla 7 se encuentran recogidos los datos referentes a la frecuencia de las diferentes categorías de actividad distinguiendo por posición habitual de juego en cada una de las competiciones así como en baloncesto femenino en su conjunto. Si se observa la frecuencia total de las categorías de actividad no se detecta la existencia de diferencias significativas entre posiciones en ninguna de las competiciones, ni en el baloncesto femenino en su conjunto. Sin embargo, si se analiza cada una de las categorías de actividad por separado sí que se detectan diferencias significativas que parecen seguir un patrón común en ambas competiciones. Así en las categorías parado/caminando, shuffle baja intensidad y shuffle media intensidad las diferencias se localizan entre bases por un lado y aleros y pívots por el otro, siendo inferior la frecuencia en bases. Por otro lado, en las categorías sprint y shuffle alta intensidad las diferencias vuelven a localizarse de la misma manera, pero en este caso las jugadoras en la posición de base registran la mayor frecuencia en ambos casos. Finalmente encontramos también diferencias en la categoría salto, donde los aleros se diferencian de manera significativa tanto de bases como de pívots. Si atendemos al baloncesto femenino en su conjunto, las diferencias que aparecen siguen un patrón similar a lo ya señalado para cada una de las competiciones por separado.

En la tabla 8 se recogen los datos relativos a la duración media de las actividades en las diferentes categorías, donde no se detectaron diferencias estadísticamente significativas.

Finalmente, en la tabla 9 se recogen los datos referentes al porcentaje de TR en cada categoría de actividad por posiciones habituales de juego. En este caso los resultados obtenidos con ANOVA revelan la existencia de un patrón común en EF y CM y en el conjunto del baloncesto femenino. Así se encontraron diferencias estadísticamente significativas en las categorías parado/caminando, shuffle baja intensidad, sprint, y shuffle alta intensidad en ambas competiciones. Dichas diferencias se localizan entre bases por un lado y aleros y pívots por el otro. En las dos primeras categorías los registros son inferiores en las jugadoras en la posición de base y en los dos siguientes son superiores en dicha posición. Al analizar los datos obtenidos en baloncesto femenino se observa que en la posición de base las categorías que ocupan un mayor porcentaje de TR son trote y carrera, seguidos de las categorías parado/caminando, shuffle baja intensidad y shuffle media intensidad. Por otro lado las categorías que ocupan un menor porcentaje son sprint, shuffle alta intensidad y salto. En las posiciones de pívot y alero ocurre algo similar, pero en ambos casos la categoría que ocupa un mayor porcentaje de TR es parado/caminando. 
Oliveira-Da-Silva, L.; Sedano-Campo, S.; Redondo-Castán, J.C. (2013). Características del esfuerzo en competición en jugadoras de baloncesto de élite durante las fases finales de la Euroliga y el Campeonato del Mundo. RICYDE. Rev. int. cienc. deporte. 34(9), 360-376. http://dx.doi.org/10.5232/ricyde2013.03405

Tabla 7: Frecuencia de las diferentes categorías de actividad distinguiendo por posición habitual de juego en cada competición y en el conjunto del baloncesto femenino.

\begin{tabular}{|c|c|c|c|c|c|c|c|c|c|}
\hline & \multicolumn{5}{|c|}{ Acciones de baja intensidad } & \multicolumn{3}{|c|}{ Acciones de alta intensidad } & \multirow[b]{2}{*}{ Total } \\
\hline Euroliga femenina & $\begin{array}{c}\text { Parado/ } \\
\text { caminando }\end{array}$ & Trote & Carrera & $\begin{array}{l}\text { Shuffle baja } \\
\text { intensidad }\end{array}$ & $\begin{array}{c}\text { Shuffle media } \\
\text { intensidad }\end{array}$ & $\begin{array}{l}\text { Shuffle alta } \\
\text { intensidad }\end{array}$ & Sprint & Salto & \\
\hline Base & $122 \pm 9.10_{\mathrm{a}}$ & $191 \pm 7.61$ & $173 \pm 11.11$ & $130 \pm 11.30_{\mathrm{a}}$ & $141 \pm 10.11_{\mathrm{a}}$ & $82 \pm 3.40_{a}$ & $94 \pm 6.11_{\mathrm{a}}$ & $19 \pm 2.60_{\mathrm{a}}$ & $952 \pm 7.51$ \\
\hline Pívot & $150 \pm 12.30_{b}$ & $183 \pm 4.22$ & $160 \pm 10.31$ & $216 \pm 9.24_{b}$ & $204 \pm 10.23_{b}$ & $50 \pm 4.71_{b}$ & $50 \pm 4.21_{b}$ & $23 \pm 5.22_{a}$ & $1036 \pm 6.92$ \\
\hline Alero & $189 \pm 8.10_{b}$ & $175 \pm 7.80$ & $159 \pm 8.11$ & $233 \pm 12.10_{b}$ & $183 \pm 8.32_{b}$ & $56 \pm 2.16_{b}$ & $45 \pm 2.31_{b}$ & $38 \pm 3.92_{b}$ & $1078 \pm 6.41$ \\
\hline$F$ & 9.12 & .22 & .10 & 8.78 & 6.78 & 11.02 & 7.18 & 4.26 & 5.12 \\
\hline$p$ & $.003^{*}$ & .95 & .73 & $.002^{*}$ & $.001^{*}$ & $.001^{*}$ & $.001^{*}$ & $.004^{*}$ & .006 \\
\hline \multicolumn{10}{|l|}{$\begin{array}{c}\text { Campeonato del } \\
\text { Mundo }\end{array}$} \\
\hline Base & $122 \pm 8.31_{\mathrm{a}}$ & $186 \pm 9.21$ & $142 \pm 5.80$ & $129 \pm 9.12_{a}$ & $124 \pm 8.24 \mathrm{a}$ & $67 \pm 3.10_{\mathrm{a}}$ & $85 \pm 3.23_{a}$ & $10 \pm 1.84_{a}$ & $865 \pm 5.80$ \\
\hline Pívot & $142 \pm 5.70_{b}$ & $165 \pm 9.72$ & $144 \pm 10.21$ & $183 \pm 10.23_{b}$ & $138 \pm 7.51_{b}$ & $29 \pm 4.11_{b}$ & $49 \pm 5.04_{b}$ & $15 \pm 2.74_{a}$ & $865 \pm 7.91$ \\
\hline Alero & $175 \pm 10.21_{b}$ & $148 \pm 11.11$ & $116 \pm 8.60$ & $170 \pm 8.72_{b}$ & $140 \pm 6.32_{b}$ & $36 \pm 2.21_{b}$ & $38 \pm 4.83_{b}$ & $22 \pm 6.53_{b}$ & $845 \pm 8.42$ \\
\hline$F$ & 5.45 & 1.17 & .93 & 7.78 & 1.44 & 5.22 & 6.43 & 4.22 & 1.44 \\
\hline$p$ & $.01^{*}$ & .36 & .56 & $.003^{*}$ & $.04^{*}$ & $.01^{*}$ & $.01^{*}$ & $.04^{*}$ & .64 \\
\hline \multicolumn{10}{|l|}{ Baloncesto femenino } \\
\hline Base & $122 \pm 8.41_{\mathrm{a}}$ & $188.5 \pm 8.55$ & $157.5 \pm 4.76$ & $129.5 \pm 9.15_{a}$ & $132.5 \pm 10.15_{a}$ & $74.5 \pm 3.25_{a}$ & $89.5 \pm 10.29_{a}$ & $14.5 \pm 2.20_{\mathrm{a}}$ & $908.5 \pm 6.72$ \\
\hline Pívot & $146 \pm 92_{b}$ & $174 \pm 7.01$ & $152 \pm 10.21$ & $199.5 \pm 19.40_{b}$ & $171 \pm 8.94_{b}$ & $39.5 \pm 4.41_{b}$ & $49.5 \pm 4.61_{b}$ & $19 \pm 40_{a}$ & $950.5 \pm 7.43$ \\
\hline Alero & $182 \pm 9.15_{b}$ & $161.5 \pm 9.51$ & $137.5 \pm 8.35$ & $201.5 \pm 10.41_{b}$ & $161.5 \pm 7.38_{b}$ & $46 \pm 2.15_{b}$ & $41.5 \pm 3.55_{b}$ & $30 \pm 5.21_{b}$ & $961.5 \pm 7.42$ \\
\hline$F$ & 7.16 & 1.39 & .12 & 10.22 & 6.45 & 7.33 & 5.50 & 5.12 & .99 \\
\hline$p$ & $.01^{\star}$ & .17 & .99 & $.001^{*}$ & $.04^{*}$ & $.01^{*}$ & $.002^{*}$ & $.04^{*}$ & .84 \\
\hline
\end{tabular}

*p<0.05.

Los datos en la misma columna con distinto subíndice presentan diferencias estadísticamente significativas 
Oliveira-Da-Silva, L.; Sedano-Campo, S.; Redondo-Castán, J.C. (2013). Características del esfuerzo en competición en jugadoras de baloncesto de élite durante las fases finales de la Euroliga y el Campeonato del Mundo. RICYDE. Rev. int. cienc. deporte. 34(9), 360-376. http://dx.doi.org/10.5232/ricyde2013.03405

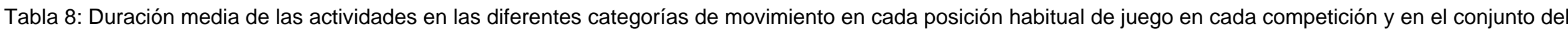
baloncesto femenino.

\begin{tabular}{|c|c|c|c|c|c|c|c|c|c|}
\hline \multirow[b]{2}{*}{$\begin{array}{c}\text { Euroliga } \\
\text { femenina }\end{array}$} & \multicolumn{5}{|c|}{ Acciones de baja intensidad } & \multicolumn{3}{|c|}{ Acciones de alta intensidad } & \multirow[b]{2}{*}{ Media } \\
\hline & Parado/caminando & Trote & Carrera & $\begin{array}{c}\text { Shuffle baja } \\
\text { intensidad }\end{array}$ & $\begin{array}{c}\text { Shuffle media } \\
\text { intensidad }\end{array}$ & $\begin{array}{c}\text { Shuffle alta } \\
\text { intensidad }\end{array}$ & Sprint & Salto & \\
\hline Base & $2.10 \pm .11$ & $1.81 \pm .05$ & $1.80 \pm .10$ & $1.60 \pm .11$ & $1.70 \pm .12$ & $1.62 \pm .12$ & $1.37 \pm .06$ & $0.77 \pm .03$ & $1.57 \pm .08$ \\
\hline Pívot & $2.01 \pm .11$ & $1.81 \pm .06$ & $1.81 \pm .08$ & $1.60 \pm .09$ & $1.81 \pm .14$ & $1.84 \pm .12$ & $1.41 \pm .07$ & $0.81 \pm .05$ & $1.62 \pm .08$ \\
\hline Alero & $2.20 \pm .10$ & $1.70 \pm .05$ & $1.91 \pm .07$ & $1.70 \pm .11$ & $1.81 \pm .12$ & $1.71 \pm .13$ & $1.36 \pm .06$ & $.62 \pm .06$ & $1.61 \pm .09$ \\
\hline$F$ & .62 & .74 & .10 & .61 & .83 & 1.02 & .78 & 1.06 & 1.12 \\
\hline$p$ & .95 & .45 & .73 & .13 & .22 & .87 & .89 & .53 & .65 \\
\hline \multicolumn{10}{|c|}{$\begin{array}{c}\text { Campeonato } \\
\text { del Mundo }\end{array}$} \\
\hline Base & $2.2 \pm .11$ & $1.90 \pm .06$ & $1.91 \pm .11$ & $1.70 \pm .10$ & $1.90 \pm .12$ & $2.01 \pm .10$ & $1.41 \pm .07$ & $.80 \pm .06$ & $1.72 \pm .09$ \\
\hline Pívot & $2.2 \pm .15$ & $1.85 \pm .07$ & $1.83 \pm .08$ & $1.66 \pm .08$ & $1.84 \pm .09$ & $1.93 \pm .12$ & $1.42 \pm .08$ & $.80 \pm .05$ & $1.66 \pm .09$ \\
\hline Alero & $2.0 \pm .12$ & $1.84 \pm .04$ & $1.83 \pm .09$ & $1.71 \pm .12$ & $1.84 \pm .10$ & $1.87 \pm .11$ & $1.41 \pm .06$ & $.74 \pm .03$ & $1.62 \pm .08$ \\
\hline$F$ & 1.445 & 1.00 & .93 & .11 & .44 & .27 & 1.13 & 1.02 & 1.00 \\
\hline$p$ & .09 & .78 & .56 & .39 & .19 & .47 & .76 & .15 & .73 \\
\hline \multicolumn{10}{|l|}{$\begin{array}{c}\text { Baloncesto } \\
\text { femenino }\end{array}$} \\
\hline Base & $2.15 \pm .11$ & $1.85 \pm .05$ & $1.85 \pm .10$ & $1.65 \pm .11$ & $1.80 \pm .11$ & $1.80 \pm .11$ & $1.35 \pm .06$ & $.75 \pm .04$ & $1.65 \pm .08$ \\
\hline Pívot & $2.11 \pm .13$ & $1.80 \pm .06$ & $1.80 \pm .08$ & $1.60 \pm .08$ & $1.80 \pm .09$ & $1.85 \pm .12$ & $1.40 \pm .07$ & $.80 \pm .05$ & $1.64 \pm .08$ \\
\hline Alero & $2.11 \pm .11$ & $1.75 \pm .04$ & $1.85 \pm .08$ & $1.70 \pm .11$ & $1.80 \pm .11$ & $1.75 \pm .12$ & $1.35 \pm .06$ & $.65 \pm .05$ & $1.61 \pm .08$ \\
\hline$F$ & .33 & 1.05 & .12 & .72 & .16 & .44 & .43 & .12 & .67 \\
\hline$p$ & .19 & .68 & .99 & .11 & .22 & .68 & .74 & .15 & .36 \\
\hline
\end{tabular}

${ }^{*} \mathrm{p}<0.05$.

Los datos en la misma columna con distinto subíndice presentan diferencias estadísticamente significativas 
Oliveira-Da-Silva, L.; Sedano-Campo, S.; Redondo-Castán, J.C. (2013). Características del esfuerzo en competición en jugadoras de baloncesto de élite durante las fases finales de la Euroliga y el Campeonato del Mundo. RICYDE. Rev. int. cienc. deporte. 34(9), 360-376. http://dx.doi.org/10.5232/ricyde2013.03405

Tabla 9: Porcentaje de Tiempo Real empleado en cada categoría por actividad en cada posición habitual de juego en cada competición y en el conjunto del baloncesto femenino.

\begin{tabular}{|c|c|c|c|c|c|c|c|c|}
\hline & \multicolumn{5}{|c|}{ Acciones de baja intensidad } & \multicolumn{3}{|c|}{ Acciones de alta intensidad } \\
\hline Euroliga femenina & Parado/caminando & Trote & Carrera & $\begin{array}{c}\text { Shuffle baja } \\
\text { intensidad }\end{array}$ & $\begin{array}{c}\text { Shuffle media } \\
\text { intensidad }\end{array}$ & $\begin{array}{l}\text { Shuffle alta } \\
\text { intensidad }\end{array}$ & Sprint & Salto \\
\hline Base & $17 \pm .55_{\mathrm{a}}$ & $19 \pm 2.10$ & $18 \pm 2.31$ & $13 \pm 1.10_{\mathrm{a}}$ & $15 \pm 2.10$ & $8 \pm 1.13_{a}$ & $9 \pm .31_{\mathrm{a}}$ & $.80 \pm .21$ \\
\hline Pívot & $20 \pm 1.01_{b}$ & $18 \pm 1.71$ & $18 \pm 1.84$ & $15 \pm 1.02_{b}$ & $14 \pm 2.22$ & $7 \pm .74_{b}$ & $7 \pm .60_{\mathrm{b}}$ & $.90 \pm .31$ \\
\hline Alero & $21 \pm .90_{b}$ & $19 \pm 1.13$ & $18 \pm 1.22$ & $14.50 \pm .91_{b}$ & $14 \pm 1.81$ & $6 \pm .62_{b}$ & $6.50 \pm .45_{b}$ & $1 \pm .44$ \\
\hline$F$ & 7.13 & .64 & .10 & 6.44 & .26 & 3.02 & 5.62 & 1,71 \\
\hline$p$ & $.01^{*}$ & .68 & .99 & $.04^{*}$ & .44 & $.04^{*}$ & $.05^{*}$ & .10 \\
\hline \multicolumn{9}{|l|}{$\begin{array}{c}\text { Campeonato del } \\
\text { mundo }\end{array}$} \\
\hline Base & $18.50 \pm 1.11_{\mathrm{a}}$ & $18.50 \pm 1.60$ & $18 \pm 1.42$ & $13 \pm .91_{\mathrm{a}}$ & $14 \pm 2.12$ & $8 \pm .72 a$ & $9 \pm .72_{a}$ & $.81 \pm .62$ \\
\hline Pívot & $23 \pm 1.32_{b}$ & $18 \pm 2.13$ & $17.50 \pm 1.52$ & $14.50 \pm .83_{\mathrm{b}}$ & $13.5 \pm 1.90$ & $6 \pm .63_{\mathrm{b}}$ & $6.51 \pm .51_{b}$ & $.81 \pm .51$ \\
\hline Alero & $22.50 \pm 1.53_{b}$ & $19 \pm 1.81$ & $17.51 \pm 2.01$ & $15 \pm .92_{b}$ & $13.5 \pm 2.13$ & $5.51 \pm .61_{b}$ & $6 \pm .62_{b}$ & $.91 \pm .40$ \\
\hline$F$ & 6.35 & 1.95 & .85 & 5.35 & .86 & 3.27 & 7.66 & 1 \\
\hline$p$ & $.02^{*}$ & .69 & .65 & $.01^{*}$ & .29 & $.04^{*}$ & $.05^{\star}$ & .45 \\
\hline \multicolumn{9}{|l|}{ Baloncesto femenino } \\
\hline Base & $17.75 \pm .80_{\mathrm{a}}$ & $18.75 \pm 1.85$ & $18 \pm 1.85$ & $13 \pm 1.01_{\mathrm{a}}$ & $14.5 \pm 2.11$ & $8 \pm .91_{a}$ & $9 \pm .52_{a}$ & $0.80 \pm .40$ \\
\hline Pívot & $21.50 \pm 1.15_{b}$ & $18 \pm 1.90$ & $18 \pm 1.65$ & $14.75 \pm .90_{b}$ & $13.75 \pm 2.05$ & $6.5 \pm .65_{b}$ & $6.75 \pm .55_{b}$ & $0.85 \pm .40$ \\
\hline Alero & $21.75 \pm 1.20_{b}$ & $19 \pm 1.45$ & $1.85 \pm 1.60$ & $14.75 \pm .90_{b}$ & $13.75 \pm 1.95$ & $5.75 \pm .60_{b}$ & $6.25 \pm .52_{b}$ & $0.95 \pm .41$ \\
\hline$F$ & 6.57 & .99 & .44 & 3.35 & .50 & 4.74 & 5.53 & .55 \\
\hline$p$ & $.01^{*}$ & .55 & .85 & $.03^{*}$ & .47 & $.01^{*}$ & $.04^{*}$ & .79 \\
\hline
\end{tabular}

Los datos en la misma columna con distinto subíndice presentan diferencias estadísticamente significativas 
Oliveira-Da-Silva, L.; Sedano-Campo, S.; Redondo-Castán, J.C. (2013). Características del esfuerzo en competición en jugadoras de baloncesto de élite durante las fases finales de la Euroliga y el Campeonato del Mundo. RICYDE. Rev. int. cienc. deporte. 34(9), 360-376. http://dx.doi.org/10.5232/ricyde2013.03405

\section{Discusión}

Debido a la escasez de datos referentes a los patrones de movimiento en baloncesto femenino, el principal objetivo del presente estudio fue analizar las características del esfuerzo en jugadoras de alto nivel. Con el objetivo de buscar una muestra representativa se analizaron esas características dentro de la fase final de dos de las principales competiciones femeninas a nivel mundial, la Euroliga y el Campeonato del Mundo, utilizando una muestra total de noventa y seis jugadoras que, en términos de edad y perfil antropométrico, puede considerarse característica del baloncesto femenino de élite (Carter, Ackland, Kerr y Stapff, 2005; Rodríguez-Alonso y col., 2003). Teniendo en cuenta las hipótesis planteadas al inicio del estudio, los resultados corroboran el carácter intermitente de esta modalidad deportiva así como la existencia de diferencias en el patrón de esfuerzo entre competiciones y entre posiciones.

Como ya se ha señalado, los resultados obtenidos confirman la naturaleza híbrida de este deporte en el que se alternan esfuerzos aeróbicos y anaeróbicos. En este sentido, las actividades predominantes durante un partido tienen carácter aeróbico (parado/caminando, trote, carrera y shuffle baja y media intensidad), ocupando éstas un porcentaje total aproximado del $85 \%$, mientras que las actividades que pudiéramos considerar de alta intensidad o anaeróbicas (sprint, shuffle alta intensidad y salto) ocupan el 15\% restante. Esta naturaleza híbrida ya ha sido mencionada por otros autores en estudios anteriores efectuados con muestras de jugadores jóvenes (Abdelkrim y col., 2007), jugadores adultos de élite (McIness y col., 1995), jugadores adultos pero no de élite (Narazaki y col., 2009) e incluso en jugadores mayores (Tessitore y col., 2006). En todos estos estudios la mayor parte del tiempo está dedicado a actividades de baja intensidad, aunque todos los autores coinciden en afirmar que son las acciones de alta intensidad las que determinan el rendimiento final. En este sentido, autores como Boyle y col. (1994) indicaban que ese patrón de esfuerzo híbrido es común en distintos deportes colectivos entre los que se incluye el baloncesto, el fútbol o el hockey.

La frecuencia total de actividades registrada en el presente estudio es ligeramente superior a la señalada por Abdelkrim y col. (2007) pero inferior a la de McInnes y col. (1995). Por otro lado, la duración media de las acciones no supera los 2s, algo que coincide con lo señalado por esos autores, aunque es inferior a lo señalado por Narazaki y col. (2009) en una muestra de menor nivel competitivo. De la misma manera, en la muestra analizada se produce un cambio de actividad aproximadamente cada 2 segundos, lo que coincide con lo señalado por McInnes y col. (1995) y Abdelkrim y col. (2007). Por lo tanto, la alta frecuencia de actividades que se registra, así como su baja duración media ilustra la idea de que el baloncesto femenino, al menos en estas categorías, también es un deporte con multitud de cambios en la intensidad y en el tipo de desplazamiento. El hecho de que la frecuencia sea incluso superior a la obtenida por McInnes y col. (1995) en jugadores varones de élite puede estar vinculado a las modificaciones reglamentarias impuestas a partir del año 2000, que implican ataques más rápidos por un menor tiempo de posesión del balón.

El hecho de que esas acciones de carácter anaeróbico ocupen un menor porcentaje del tiempo real de juego no quiere decir que sean menos importantes, puesto que tal y como se ha señalado (McIness y col., 1995; Tessitore y col., 2006) son determinantes en situaciones vitales para el rendimiento como los contraataques, la recuperación defensiva, los lanzamientos o las acciones individuales de ataque frente a la canasta. Consecuentemente, mejorar la capacidad de las jugadoras para realizar esas acciones a la máxima intensidad es fundamental para incrementar el rendimiento final (McIness y col., 1995). Por otro lado, el 
Oliveira-Da-Silva, L.; Sedano-Campo, S.; Redondo-Castán, J.C. (2013). Características del esfuerzo en competición en jugadoras de baloncesto de élite durante las fases finales de la Euroliga y el Campeonato del Mundo. RICYDE. Rev. int. cienc. deporte. 34(9), 360-376. http://dx.doi.org/10.5232/ricyde2013.03405

hecho de que las acciones de menor intensidad ocupen un mayor porcentaje de tiempo, resulta lógico si tenemos en cuenta que son las acciones más empleadas cuando se efectúa un ataque o una defensa de carácter posicional así como cuando se produce una transición ataque-defensa que no implique tanta urgencia como el contraataque.

Tal y como afirman Rodríguez-Alonso y col. (2003), Abdelkrim y col. (2007) y Narazaki y col. (2009) ese patrón intermitente al que venimos haciendo referencia tiene un reflejo claro en la carga fisiológica y metabólica a la que se ve sometido el jugador durante la competición, según se extrae de los datos relativos a frecuencia cardiaca media, a acumulación de lactato o a consumo de oxígeno. La alta acumulación de lactato así como la elevada frecuencia cardiaca media pone en evidencia la importante demanda fisiológica impuesta sobre las jugadoras de baloncesto durante la competición, independientemente de que el porcentaje de tiempo ocupado por las actividades de alta intensidad sea pequeño. En la misma línea Narazaki y col. (2009) afirman que el baloncesto competitivo requiere de una importante utilización del metabolismo aeróbico, pero son los fosfágenos la fuente energética fundamental en esas acciones cortas de alta intensidad. Dicha vía metabólica tiene que ser restaurada continuamente durante la práctica, cobrando especial importancia en este sentido la vía aeróbica. Por su parte, Sallet y col. (2005) indican que, si bien existe bastante homogeneidad en las demandas aeróbicas al comparar distintos niveles competitivos, la capacidad anaeróbica parece ser un mejor predictor del rendimiento.

Aunque se confirma el carácter intermitente del baloncesto a este nivel, es necesario remarcar la existencia de diferencias significativas cuando comparamos las dos competiciones estudiadas, a pesar de que ambas pueden considerarse competiciones de alto nivel. En primer lugar, se observa que aunque el tiempo total en el que las jugadoras permanecen en la cancha es similar en ambas competiciones, no lo es el tiempo real de juego. De hecho, los partidos de Euroliga, disputados por clubes, implican un mayor tiempo real absoluto así como un mayor porcentaje de tiempo real respecto al tiempo total de permanencia en cancha, lo que significa que, al menos en términos de volumen, la exigencia en jugadoras de Euroliga es superior. El porcentaje de TR obtenido en esta competición se aproxima mucho al registrado por Abdelkrim y col. (2007) en jugadores jóvenes, aunque es inferior al registrado por McInnes y col. (1995) en jugadores profesionales. Por otro lado, en jugadoras de Euroliga se observa un mayor porcentaje de tiempo ocupado por acciones de carácter anaeróbico. Por su parte, las acciones de menor intensidad son mayores en la competición por selecciones. Se observa por tanto que si bien antes hablábamos de una mayor exigencia en jugadoras de Euroliga en términos de volumen, también se confirma una mayor exigencia en términos de intensidad. Rodríguez-Alonso y col. (2003) también evalúan a mujeres jugadoras de alto nivel (Selección Olímpica y Primera División Nacional), señalando que las demandas fisiológicas y metabólicas competitivas en términos de intensidad son superiores a medida que se incrementa el nivel competitivo. En su caso la competición por selecciones tiene mayores exigencias que la Primera División Nacional.

Cuando se analizan las características del esfuerzo en función de la posición habitual de juego, nos encontramos con un patrón muy similar en ambas competiciones y en el baloncesto femenino en su conjunto. En este sentido, se observa que sólo las jugadoras en la posición de base presentan un perfil específico de esfuerzo que las diferencia de aleros y pívots, ya que éste se caracteriza por una menor frecuencia de actividades de baja intensidad por un lado y una mayor frecuencia de las acciones de mayor intensidad. Esto está en línea con lo señalado por Abdelkrim y col. (2007) y puede deberse a que los jugadores en esa posición son por lo general más activos, ya que están normalmente implicados en la posesión 
Oliveira-Da-Silva, L.; Sedano-Campo, S.; Redondo-Castán, J.C. (2013). Características del esfuerzo en competición en jugadoras de baloncesto de élite durante las fases finales de la Euroliga y el Campeonato del Mundo. RICYDE. Rev. int. cienc. deporte. 34(9), 360-376. http://dx.doi.org/10.5232/ricyde2013.03405

del balón y en las rápidas transiciones entre ataque y defensa, realizando mayor cantidad de acciones de alta intensidad que los pívots y aleros. Estas diferencias en el patrón de esfuerzo también tienen su reflejo en la carga fisiológica y metabólica impuesta sobre las jugadoras en posición de base, respecto a pívots y aleros (Rodríguez-Alonso y col., 2003), siendo superior en las primeras. Cabe destacar por otro lado, el hecho de que la acción salto parece ser más importante en términos cuantitativos en la posición de alero, algo que podemos vincular al rol técnico-táctico que esta posición desempeña durante la competición (Abdelkrim y col., 2007).

\section{Conclusiones}

Como conclusión es necesario señalar que el presente estudio confirma el carácter intermitente y acíclico del baloncesto femenino en ambas competiciones. En este sentido el tipo de competición analizada tiene una influencia notable en el tiempo ocupado por las acciones de alta intensidad, siendo éste superior en los partidos de Euroliga Femenina, por lo que puede afirmarse que en esta competición la exigencia del esfuerzo en términos de intensidad es superior. Especialmente interesantes son los hallazgos relativos al perfil específico de esfuerzo que se aprecia en las jugadoras en posición de base en ambas competiciones, con unas mayores exigencias fisiológicas cuando las comparamos con el resto de posiciones. Tales hallazgos son importantes de cara a la planificación e individualización de los entrenamientos por parte de los entrenadores y preparadores físicos en estas categorías, ya que conocer las características específicas del esfuerzo al que es sometido un deportista durante la competición es fundamental para el diseño de las tareas de entrenamiento. En esta misma línea, la utilización de acciones específicas de baloncesto, adaptadas en intensidad y volumen a las exigencias reales, como medio de entrenamiento garantiza la especificidad del estímulo. No obstante, los resultados aquí obtenidos no pretenden ser generalizables a todo el baloncesto femenino, sino que son sólo un primer paso en el análisis de las demandas competitivas de este deporte, análisis centrado específicamente en dos competiciones. Es preciso efectuar estudios similares que analicen el patrón de esfuerzo en otras categorías competitivas. Sólo de esa manera podremos conocer las características reales de esfuerzo en cada caso y a partir de ahí diseñar los distintos protocolos de entrenamiento. 
Oliveira-Da-Silva, L.; Sedano-Campo, S.; Redondo-Castán, J.C. (2013). Características del esfuerzo en competición en jugadoras de baloncesto de élite durante las fases finales de la Euroliga y el Campeonato del Mundo. RICYDE. Rev. int. cienc. deporte. 34(9), 360-376. http://dx.doi.org/10.5232/ricyde2013.03405

\section{Referencias}

Abdelkrim, B.N.; El Fazaa, S., \& El Ati, J. (2007). Time-motion analysis and physiological data of elite under-19 basketball players during competition. British Journal of Sports Medicine, 41, 69-75.

Anguera, M.T. (1990). Metodología observacional. En J. Arnau, M.T. Anguera y J. Gómez (Eds.), Metodología de la investigación en Ciencias del Comportamiento (pp.125236). Murcia: Secretariado de Publicaciones de la Universidad de Murcia.

Anguera, M.T.; Blanco-Villaseñor, A.; Losada, J.L., y Sánchez Algarra, P. (1999). Análisis de la competencia en la selección de observadores. Metodología de las Ciencias del Comportamiento, 1(1), 95-115.

Bompa, T. O., \& Haff, G.G. (2009). Periodization. Theory and Methodology of Training (5th ed.). Champaign, IL: Human Kinetics.

Boyle, P.M.; Mahoney, C.A., \& Wallace, W.F.M. (1994). The competitive demands of elite male field hockey. Journal of Sports Medicine and Physical Fitness, 34, 235-241.

Carter, J.E.L.; Ackland ,T.R.; Kerr, D.A., \& Stapff A.B. (2005). Somatotype and size of elite female basketball players. Journal of Sports Sciences, 23(10), 1057-1063.

Castagna, C.; D’Ottavio S.; Manzi, V.; Annino, G.; Colli, R.; Belardinelli, R., \& Lacalaprice, F. (2005). HR and $\mathrm{VO}_{2}$ responses during basketball drills. En N. Dikic, S. Zinanic, S. Astojic \& Z. Tornjanski (Eds.), Book of Abstracts of the 10th Annual Congress of European College of Sport Science (pp. 160). Belgrade: European College of Sport Science.

Chaouachi, A.; Brughelli, M.; Chamari, K.; Levin, G.T.; Abdelkrim, N.; Laurencelle, L., \& Castagna, C. (2009). Lower limb maximal dynamic strength and agility determinants in elite basketball players. Journal of Strength and Conditioning Research, 23, 15701577.

Drinkwater, E.J.; Pyne, D.B., \& McKenna, M.J. (2008). Design and interpretation of anthropometric and fitness testing of basketball players. Sports Medicine, 38, 565578.

Hoffman, J.R., \& Maresh, C.M. (2000). Physiology of basketball. En W.E. Garret \& D.T. Kirkendall (Eds), Exercise and sports science (pp. 733-744). Philadelphia: Lippincott Williams and Wilkins.

Gil, A., y Contreras, J. (2008) Los porteros de fútbol. ¿Se comportan como sistemas complejos?: estudio de Iker Casillas y Víctor Valdés. Barcelona. Universidad de Barcelona.

Medina, J., y Delgado, M.A. (1999). Metodología de entrenamiento de observadores para investigaciones sobre Educación Física y Deporte en las que se utilice como método la observación. Motricidad. European J ournal of Human Movement, 5, 69-86.

Mclnnes, S.E.; Carlson, J.S.; Jones, C.J., \& McKenna, M.J. (1995). The physiological load imposed on basketball players during competition. Journal of Sports Sciences, 13(5), 387-397.

Miller, S.A., \& Barlett, R.M. (1994). Notational analysis of the physical demands of basketball. J ournal of Sports Sciences, 12, 181.

Naclerio, F. (2011). Entrenamiento deportivo. Madrid: Médica Panamericana.

Narazaki, K., \& Berg, K. (2006). Bioenergetics and time-motion analysis of competitive basketball. Medicine and Science in Sports and exercise, 38, 238-239. 
Oliveira-Da-Silva, L.; Sedano-Campo, S.; Redondo-Castán, J.C. (2013). Características del esfuerzo en competición en jugadoras de baloncesto de élite durante las fases finales de la Euroliga y el Campeonato del Mundo. RICYDE. Rev. int. cienc. deporte. 34(9), 360-376. http://dx.doi.org/10.5232/ricyde2013.03405

Narazaki, K.; Berg, K.; Stergiou, N., \& Chen, B. (2009). Physiological demands of competitive basketball. Scandinavian Journal of Medicine and Science in Sports, 19, $425-432$.

Ostojic, S.M.; Mazic, S., \& Dikic, N. (2006). Profiling in basketball: physical and physiological characteristics of elite players. Journal of Strength and Conditioning Research, 20(4), 740-744.

Rodriguez-Alonso, M.; Fernandez-Garcia, J.; Pérez-Landaluce, J., \& Terrados, N. (2003). Blood lactate and heart rate during national and international women's basketball. The J ournal of Sports Medicine and Physical Fitness, 43, 432-436.

Sallet, P.; Perrier, D.; Ferret, J.M.; Vitelli ,V., \& Baverel, G. (2005). Physiological differences in professional basketball players as a function of playing position and level of play. The J ournal of Sports Medicine and Physical Fitness, 45, 291-294.

Taylor, J. (2003). Basketball: applying time motion data to conditioning. Strength Conditioning J ournal, 25, 57-64.

Tessitore, A.; Tiberi, M.; Cortis, C.; Rapisarda, E.; Meeusen, R., \& Capranica, L. (2006). Aerobic-anaerobic profiles, heart rate and match analysis in old basketball players. Gerontology, 52, 214-222.

Ziv, G., \& Lidor, R. (2009). Vertical jump in female and male basketball players-A review of observational and experimental studies. Journal of Science and Medicine in Sports, 13(3), 332-9. 